\title{
MEJORAMIENTO ENERGÉTICO Y ECONOMICO DE UNA REFRIGERADORA DOMÉSTICA SUSTITUYENDO SU REFRIGERANTE ORIGINAL R12 POR OTRO ECOLÓGICO - ANALISIS EXPERIMENTAL
}

\author{
ENERGY AND ECONOMIC IMPROVEMENT OF DOMESTIC \\ REFRIGERATOR ONLY REPLACING ORIGINAL R12 \\ REFRIGERANT BY OTHER ECOLOGICAL REFRIGERANT - \\ EXPERIMENTAL ANALYSIS
}

\author{
Salome Gonzales Chávez ${ }^{1}$, Néstor Rosas Martínez ${ }^{2}$
}

\begin{abstract}
RESUMEN
Se demuestra de forma experimental la mejora energética y económica de una refrigeradora tipo doméstica, a partir de solamente sustituir su refrigerante original $R-12$ (CFC de característica contaminante y degradante de la Capa de Ozono), por otro refrigerante del tipo ecológico (HFC) como el R-134a. Comúnmente se practica el reemplazo de una refrigeradora que usa CFC, por otra unidad nueva que utiliza refrigerante ecológico de origen. Sin embargo, en países en vías de desarrollo el sector residencial mayormente cuenta con refrigeradoras que operan con R-12 y con vida de uso superior a los 10 años, no obstante la existencia de acuerdos internacionales que prohíben su utilización. El experimento consistió en: 1) preparación del equipo de refrigeración (dotación de reguladores de carga en el condensador y evaporador, dosificadores de refrigerante y aceite de lubricación-fluidización e instrumentación), 2) protocolo de mediciones, desarrollo de pruebas de operación con $R-12$ y con refrigerante ecológico sustituto $R-134^{a}$ para las diversas combinaciones de carga realizadas durante un amplio periodo de experimentación. La evaluación energética se basa en las comparaciones de los ciclos termodinámicos, balances energéticos, potencias y rendimientos, operando el sistema de refrigeración tanto con $R-12$ como con $R-134$ a. Como resultados se obtienen que el sistema de refrigeración operando con refrigerante ecológico $R-134$ a frente al refrigerante $R-12$ de origen, se logra condiciones termodinámicas estables de operación y menor consumo de potencia del compresor, para cualquier condición de carga y en amplios periodos de operación. Por tanto, una de las soluciones más convenientes desde el punto de vista socioeconómico, es la sustitución solamente del refrigerante CFC en la misma refrigeradora usada (antigua), haciendo los ajustes y apropiada dosificación del refrigerante ecológico y del aceite de lubricación-fluidización.
\end{abstract}

Palabras clave: Refrigeración doméstica, Refrigerante CFC, Refrigerante ecológico HFC, Capa de ozono, Eficiencia energética, Confiabilidad, Economía.

\section{ABSTRACT}

We demonstrate experimentally the energy and economic improvement of a domestic refrigerator, after only replace the original refrigerant $R-12$ (CFC) on the other ecological refrigerant $R-134 b$ (HFC). Commonly a refrigerator using $C F C$ is replaced with new green refrigerator. However, in developing countries the residential sector has mostly refrigerators operating with $R-12$ and life exceeding 10 years use, despite the existence of international agreements that prohibit their use.

\footnotetext{
${ }^{1}$ Dr. Docente Investigador de la Facultad de Ingeniería Mecánica, Universidad Nacional de Ingeniería, Lima-Perú, ${ }^{2}$ Msc. Docente de la Facultad de Ingeniería Mecánica, Universidad Nacional de Ingeniería, Lima-Perú.
} 
As a result we obtain that the refrigerator in evaluating operating with ecological refrigerant, compared with $R-12$ refrigerant source, stable thermodynamic conditions operation and lower power consumption compressor, for all load conditions and extended periods of operation is achieved. Therefore, one of the most convenient solutions from a socioeconomic view point, it is only replacing $C F C$ refrigerant used in the refrigerator (old), making the appropriate dosage of ecological coolant and lubrication oil-fluidization.

Key words.- Domestic refrigeration, CFC refrigerant, HFC refrigerant, Ozone layer, Energy efficiency, Reliability, Economy.

\section{INTRODUCCION}

En este trabajo de investigación experimental, realizado en el Laboratorio de Energía de la Facultad de Ingeniería Mecánica de la Universidad Nacional de Ingeniería, se obtiene una solución técnica, ecológica y subsecuente económica para la refrigeración doméstica y comercial. Consiste en la demostración experimental de la mejora energética, confiable y económica de una refrigeradora doméstica que funciona originalmente con R-12, sustituyendo tan sólo éste refrigerante (prohibido por su característica contaminante que deteriora la Capa de Ozono), con otro del tipo ecológico bajo dosificación adecuada de un aceite de lubricación-fluidificación (Ref. 1 y 2).

El objetivo se enmarca en la evaluación energética integral del funcionamiento de una refrigeradora experimental de laboratorio, con característica semejante al de una refrigeradora doméstica, operando primero con R-12 (CFC Cloro Fluoro Carbonados) y correspondiente compresor de origen, luego operando con refrigerante ecológico del tipo R-134a (HCF Hidro Fluoro Carbonado) sin sustituir al compresor original.

Como hipótesis se parte de que el comportamiento energético de la conversión de una refrigeradora que opera con refrigerante R-12, al ser sustituido sólo el refrigerante a otro de tipo ecológico (R134a), se logra una mejor performance energética de la refrigeradora, sin contaminación, bajos costos ni alteración de su vida útil.

A nivel nacional, no se han realizado trabajos específicos referidos al tema. En el ámbito internacional se tiene referencias de trabajos de investigación energética en sistemas de refrigeración domésticos, ello mediante simulación y modelización numérica (Ref. 3, 4 y 5).

\section{PLANTEAMIENTO DEL PROBLEMA, PROCEDIMIENTO Y CALCULOS}

\section{Planteamiento del problema}

El refrigerante R-12 es un compuesto químico de naturaleza contaminante, que al contacto con el medio ambiente deteriora irreversiblemente a la Capa de Ozono. La mayoría de las refrigeradoras domésticas utilizadas en el sector residencial y comercial del país, operan con este tipo de refrigerante, no obstante su prohibición internacional según acuerdos internacionales como el de Montreal (Ref. 6).

Reemplazar una refrigeradora que funciona con R12 por otra de característica ecológica y nueva, demanda altos costos que comúnmente no están al alcance económico de la mayoría de familias del sector socio-económico C, D y E. Actualmente se estima que de cada 100 familias, más del $80 \%$ poseen refrigeradoras que funcionan con R-12, incluso con vida de uso por encima de los 10 años. Por otro lado; reemplazar al compresor que opera con R-12 por otro que opera con refrigerante ecológico, también resulta costoso puesto que ello representa alrededor del $50 \%$ del costo de una refrigeradora ecológica nueva.

\section{PROCEDIMIENTO}

\section{Preparación de equipo e instrumentación}

A partir de un equipo instructivo de refrigeración de capacidad doméstica en situación de desuso, se procedió a:

- Reparación integral del evaporador y condensador.

- Calibración y ajuste de ventiladores simuladores de la variación de carga de evaporación y condensación. 
- Adaptación de tanques dosificadores de refrigerante y aceite lubricante hacia el circuito de refrigeración.

- Adaptación del sistema de expansión con tubo capilar y válvula de expansión termostática.

- Instalación de filtros y cambio total del circuito de tuberías.

- Instrumentación: Sustitución, calibración y ajuste de instrumentos de medición: termómetros, manómetros, flujómetro, vatímetro, voltímetro y amperímetro.

- Pruebas de funcionamiento y ajuste del compresor.

- Pruebas de cargas de evaporador y condensador.

- Pruebas del sistema de expansión.

- Pruebas de dosificación de aceite y tipo de refrigerante.

- Pruebas de hermeticidad y fluidización del sistema.

\section{Protocolo de mediciones y cálculos}

- Elaboración del protocolo de pruebas: formatos y organización del experimento.

- Proceso de mediciones: periodo de mediciones de variables eléctricas y térmicas, para operación del sistema con R-12 y con R-134.

- Tratamiento de la información de las pruebas experimentales de laboratorio

- Proceso de cálculo de variables objetivo para la determinación de la performance energética del sistema de refrigeración, operando con R-12 y con R-134 .

Los formatos elaborados para el protocolo de mediciones se muestran en el ANEXO. El equipo experimental de refrigeración preparado e instrumentado para el presente estudio, se esquematiza en la Figura 1, y el sistema eléctrico de energización del compresor se muestra en la Figura 2.

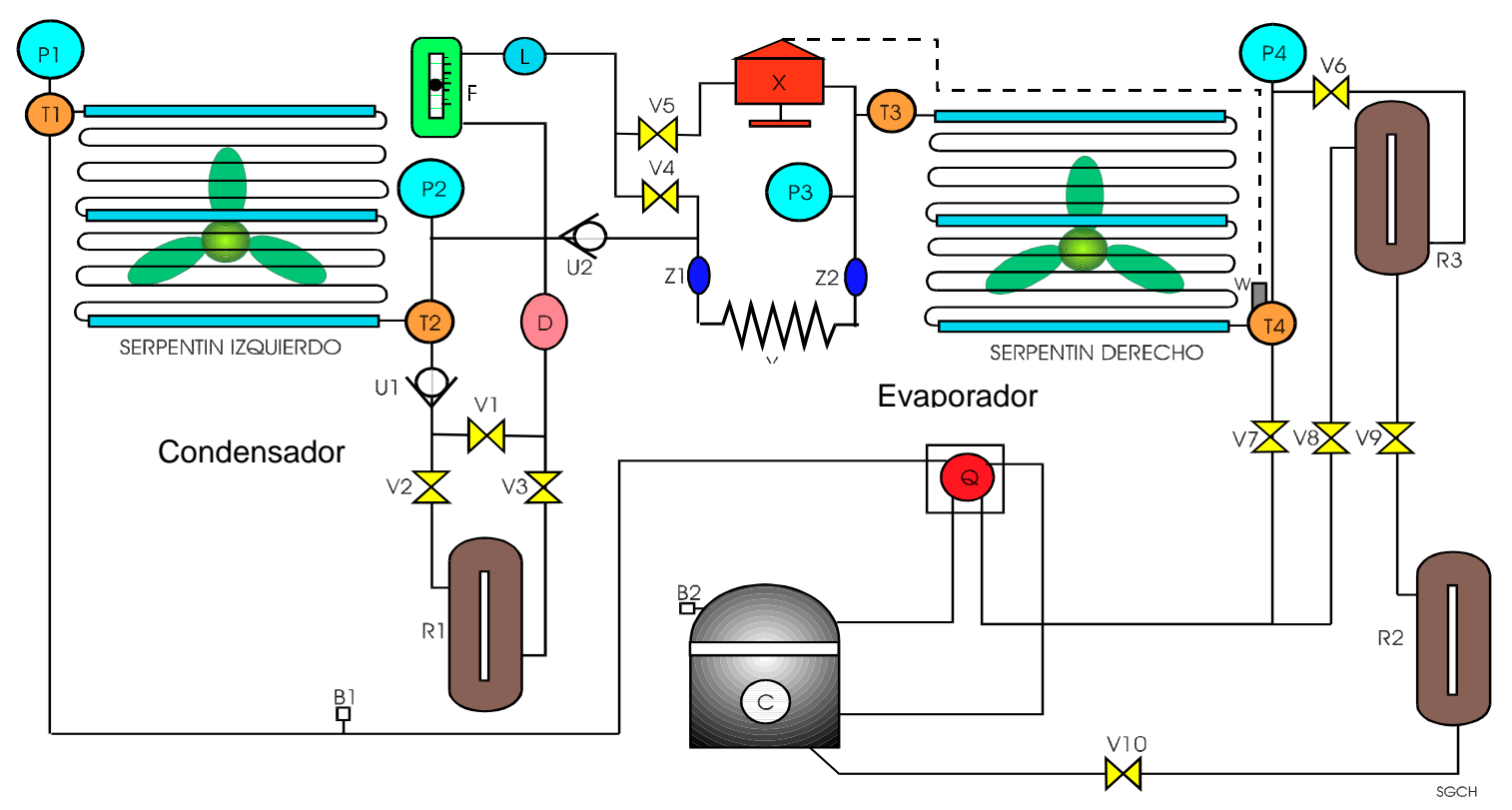

Fig. 1 Esquema del sistema experimental de refrigeración.

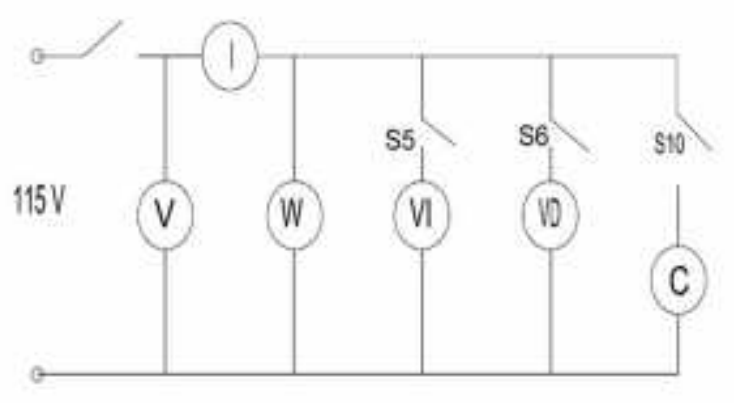

Fig. 2 Circuito eléctrico del sistema. 
Los componentes del sistema y la instrumentación se identifican mediante la siguiente notación:

C: Compresor

B: Condensador

F: Medidor de flujo

L: Indicador de líquido y humedad

D: Filtro secador (Silicagel)

Y: Tubo capilar

X: Válvula de expansión termostática

E: Evaporador

$\mathrm{R} 1$ : Dosificador de líquido refrigerante

R2: Almacenador de aceite

R3: Acumulador de aceite y refrigerante

B1, B2: Válvulas de carga y descarga de refrigerante

V1, V2:Válvulas para control de flujo de refrigerante

P1, T1: Presión y temperatura de entrada al condensador

P2, T2: Presión y temperatura de entrada al dispositivo de expansión

P3, T3: Presión y temperatura de entrada al evaporador

P4, T4: Presión y temperatura de entrada al compresor

I: Corriente eléctrica consumida por el sistema

V: Voltaje de entrada al circuito eléctrico monofásico

W: Potencia activa de entrada al sistema, medida mediante un vatímetro monofásico

\section{Pruebas de funcionamiento con R-12 y R-134}

Las pruebas experimentales del sistema de refrigeración, operando en una primera fase de experimentaciones con refrigerante R-12 $\mathrm{y}$ después operando con refrigerante $\mathrm{R}$ 134a, se han realizado utilizando dos tipos de dispositivos de expansión: una válvula termostática y un tubo capilar; ello con la finalidad de evaluar la influencia del proceso termodinámico de expansión, sobre el rendimiento y confiabilidad del evaporador, del condensador y todo el sistema de refrigeración. Las mediciones se inician una vez estabilizado el equipo para cada carga, tomando lecturas eléctricas y térmicas del sistema para las diversas condiciones de trabajo en el condensador (carga ambiente) y evaporador (carga de refrigeración), recopilados en el Formato A. En el Formato B se recogen las lecturas de las potencias de consumo y el flujo de aire de los ventiladores simuladores de carga tanto del condensador como del evaporador.

En la Figura 3 se ilustra en un diagrama presión- entalpía, el ciclo termodinámico teórico y real del sistema de refrigeración, con la notación correspondiente para el análisis energético.

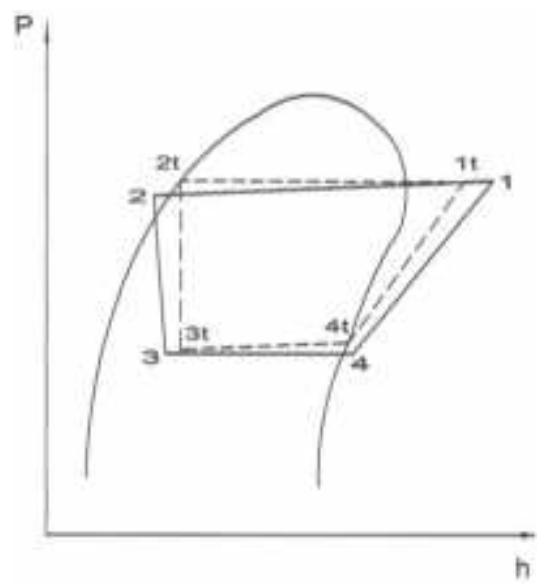

Fig. 3 Diagrama p-h teórico y real del sistema de refrigeración.

\section{CALCULOS}

\section{Potencia activa consumida por el compresor}

Es la potencia activa neta consumida por el motor del compresor, dado por:

$$
P_{A C C}=W-\left(W_{V I}+W_{V D}\right)
$$

$\mathrm{W}_{\mathrm{VI}}$ Potencia activa consumida por el ventilador izquierdo (simulador de carga en el condensador), a una determinada posición de velocidad.

$\mathrm{W}_{\mathrm{VD}}$ Potencia activa consumida por el ventilador derecho (simulador de carga en el evaporador), a una determinada posición de velocidad.

W Lectura del vatímetro.

Flujo másico de aire.- Flujo de aire impulsado por cada ventilador simulador de carga del condensador y del evaporador; calculado mediante: 


$$
\dot{m}_{\text {Aire }}=\delta_{\text {Aire }} \cdot V \cdot A_{E}
$$

$\delta_{\text {Aire }}: \quad$ Densidad del aire en el ambiente

$$
\delta_{\text {Aire }}=P_{0} / R \cdot T_{B S}
$$

V Velocidad del aire, medido con el anemómetro

$P_{0} \quad$ Presión ambiente

$\mathrm{A}_{\mathrm{E}} \quad$ Área efectiva del ventilador

TBS Temperatura de bulbo seco

Flujo calorífico del aire.- Flujo calorífico que recibe o transfiere el aire en los serpentines del condensador y evaporador (cargas). Se calcula con la siguiente expresión:

$$
\dot{Q}=\dot{m}_{\text {Aire }} \cdot C_{P-\text { aire }} \cdot\left[T_{D}-T_{P}\right]
$$

$C_{P \text {-aire }}$ Calor específico del aire a presión constante.

$T_{D} \quad$ Temperatura parte delantera del ventilador.

$T_{P} \quad$ Temperatura parte posterior del ventilador.

Potencia térmica real del compresor.-

$$
P_{T R C}=\dot{m}_{R-12} \cdot\left(h_{1}-h_{4}\right)
$$

$\dot{m}_{R-12}$ Flujo de refrigerante medido en el rotámetro.

$\mathrm{h}_{1} \quad$ Entalpía del refrigerante en la descarga del compresor.

$\mathrm{h}_{4} \quad$ Entalpía en la entrada del compresor.

Eficiencia del motor-compresor.-

$$
\eta=P_{T R C} / P_{A C C}
$$

Efecto refrigerante útil (ERU).- Se calcula con la siguiente expresión:

$$
E R U=\left(h_{4}-h_{3}\right)
$$

h4 Entalpía del flujo refrigerante en la salida del evaporador.

h3 Entalpía del refrigerante a la entrada del evaporador.
Flujo calórico útil.- Es la potencia térmica útil de refrigeración, expresado por:

$$
\dot{Q}_{U}=\dot{m}_{R-12} E R U
$$

Coeficiente de performance del ciclo.- Es el mejor indicador de la capacidad de refrigeración que se logra del ciclo termodinámico de refrigeración (Ref. 7):

$$
C O P_{\text {CICLO }}=\frac{E R U}{\left(h_{1}-h_{4}\right)}
$$

Coeficiente de performance del sistema.Representa el aprovechamiento energético total del sistema de refrigeración; es decir la razón entre la potencia o flujo calórico de refrigeración y la energía eléctrica consumida para accionar al compresor:

$$
C O P_{\text {SIST }}=\frac{\dot{Q}_{U}}{P_{A C C}}
$$

\section{PROPUESTA DE SOLUCION Y ANALISIS DE RESULTADOS}

La propuesta de solución se basa en la demostración experimental de que el sistema de refrigeración, operando con refrigerante ecológico R-134a y adecuada dosificación de aceite de lubricación, para cualquier condición de carga del evaporador y condensador, se comporte comparativamente estable y energéticamente mejor que cuando el sistema de refrigeración funciona con refrigerante R-12; todo ello sin cambiar el sistema de compresión que originalmente utiliza R-12, y con pruebas realizadas en un amplio periodo de trabajo del sistema.

Como resultado del proceso de cálculo, en la tabla 1 se muestra el comportamiento energético promedio del sistema experimental operando con refrigerante $\mathrm{R}$ 12, para las diferentes condiciones de carga del condensador y evaporador, utilizando válvula termostática y tubo capilar en el proceso de expansión. De forma análoga, en la tabla 2 se presenta el desempeño energético promedio del sistema operando con refrigerante ecológico R-134a. 
Para el análisis de resultados obtenidos, a continuación se presentan los esquemas gráficos comparativos del comportamiento energético del sistema de refrigeración operando con R-12 y con R-134a.

Tabla 1. Desempeño energético del sistema de refrigeración con refrigerante $R-12$.

\begin{tabular}{|c|c|c|c|c|c|c|c|c|}
\hline Operación & $\begin{array}{l}\text { Carga } \\
\text { Cond/Eva }\end{array}$ & $\begin{array}{l}P_{A C C} \\
(\text { watt })\end{array}$ & $\begin{array}{l}P_{T R C} \\
\text { (watt) }\end{array}$ & $\eta$ & $\begin{array}{l}E R U \\
\mathrm{k} / \mathrm{j} / \mathrm{kg}\end{array}$ & $\begin{array}{l}{ }_{0}^{+1} U \\
\text { (watt) }\end{array}$ & COP & $C O P_{\text {SIST }}$ \\
\hline \multirow{7}{*}{$\begin{array}{l}\text { Expansión } \\
\text { con Válvula } \\
\text { Termostática }\end{array}$} & $\mathrm{BB}$ & 630.0 & 297.89 & 47.28 & 117.914 & 1925.93 & 6.47 & 3.06 \\
\hline & $\mathrm{BM}$ & 665.0 & 335.05 & 50.38 & 116.901 & 1889.90 & 5.64 & 2.84 \\
\hline & $\mathrm{BA}$ & 792.5 & 354.21 & 44.69 & 116.145 & 1838.96 & 5.19 & 2.32 \\
\hline & MB & 640.0 & 408.67 & 63.86 & 120.192 & 1903.04 & 4.66 & 2.97 \\
\hline & MM & 660.0 & 393.74 & 59.66 & 120.918 & 1934.69 & 4.91 & 2.93 \\
\hline & MA & 702.5 & 382.49 & 54.45 & 120.962 & 1915.23 & 5.01 & 2.73 \\
\hline & $\mathrm{AB}$ & 552.5 & 353.09 & 63.91 & 127.132 & 1822.23 & 5.16 & 3.30 \\
\hline \multirow{11}{*}{$\begin{array}{l}\text { Expansión } \\
\text { con Tubo } \\
\text { Capilar }\end{array}$} & $\mathrm{AM}$ & 572.5 & 340.13 & 59.41 & 128.598 & 1928.97 & 5.67 & 3.37 \\
\hline & AA & 605.0 & 352.76 & 58.31 & 129.194 & 2088.64 & 5.92 & 3.45 \\
\hline & BB & 580.0 & 242.46 & 41.80 & 120.487 & 1445.84 & 5.96 & 2.49 \\
\hline & BM & 630.0 & 257.59 & 40.89 & 119.874 & 1498.43 & 5.82 & 2.38 \\
\hline & BA & 752.5 & 271.05 & 36.02 & 121.067 & 1493.16 & 5.51 & 1.98 \\
\hline & MB & 569.0 & 272.56 & 47.90 & 124.247 & 1511.67 & 5.55 & 2.66 \\
\hline & MM & 585.0 & 279.93 & 47.85 & 125.128 & 1626.66 & 5.81 & 2.78 \\
\hline & MA & 622.5 & 282.39 & 45.36 & 125.008 & 1666.77 & 5.90 & $2 . .68$ \\
\hline & $\mathrm{AB}$ & 474.5 & 238.29 & 50.25 & 133.801 & 1494.11 & 6.27 & 3.15 \\
\hline & $\mathrm{AM}$ & 492.5 & 195.46 & 39.69 & 134.228 & 1588.36 & 8.13 & 3.23 \\
\hline & AA & 505.0 & 208.80 & 41.35 & 134.811 & 1730.07 & 8.29 & 3.43 \\
\hline
\end{tabular}

Tabla 2. Performance energética del sistema de refrigeración con refrigerante $R-134^{a}$.

\begin{tabular}{|c|c|c|c|c|c|c|c|c|}
\hline Operación & $\begin{array}{c}\text { Carga } \\
\text { Cond/Eva }\end{array}$ & $\begin{array}{c}P_{A C C} \\
\text { (watt) }\end{array}$ & $\begin{array}{c}P_{T R C} \\
\text { (watt) }\end{array}$ & $\begin{array}{c}\eta \\
(\%)\end{array}$ & $\begin{array}{c}E R U \\
\mathrm{k} / \mathrm{j} / \mathrm{kg}\end{array}$ & $\begin{array}{c}\bar{\alpha} U \\
\text { (watt) }\end{array}$ & $C_{\text {CICLO }}$ & $C O P_{S I S T}$ \\
\hline \multirow{9}{*}{$\begin{array}{c}\text { Expansión } \\
\text { con } \\
\text { válvula } \\
\text { termostática }\end{array}$} & $\mathrm{BB}$ & 630.0 & 261.80 & 41.56 & 171.600 & 3775.20 & 14.42 & 5.99 \\
\hline & $\mathrm{BM}$ & 557.5 & 338.80 & 60.77 & 176.700 & 3887.40 & 11.47 & 6.97 \\
\hline & BA & 545.0 & 367.40 & 67.41 & 174.900 & 3847.80 & 10.47 & 7.06 \\
\hline & MB & 537.5 & 409.50 & 76.19 & 174.300 & 3776.50 & 9.22 & 7.03 \\
\hline & MM & 535.0 & 433.33 & 81.00 & 175.900 & 3811.17 & 8.80 & 7.12 \\
\hline & MA & 532.5 & 429.00 & 80.56 & 175.200 & 3796.00 & 8.85 & 7.13 \\
\hline & $\mathrm{AB}$ & 525.0 & 422.00 & 80.38 & 174.420 & 3488.40 & 8.27 & 6.64 \\
\hline & $\mathrm{AM}$ & 532.5 & 434.35 & 81.57 & 175.500 & 3480.75 & 8.01 & 6.54 \\
\hline & AA & 540.0 & 416.50 & 77.13 & 176.200 & 3494.63 & 8.39 & 6.47 \\
\hline \multirow{9}{*}{$\begin{array}{c}\text { Expansión } \\
\text { con tubo } \\
\text { capilar }\end{array}$} & BB & 440.0 & 136.50 & 31.02 & 175.600 & 1902.33 & 13.94 & 4.32 \\
\hline & $\mathrm{BM}$ & 437.5 & 121.33 & 27.73 & 175.700 & 1903.42 & 15.69 & 4.35 \\
\hline & BA & 435.0 & 111.58 & 25.65 & 177.600 & 1924.00 & 17.24 & 4.42 \\
\hline & $\mathrm{MB}$ & 437.5 & 131.08 & 29.96 & 177.000 & 1917.50 & 14.63 & 4.38 \\
\hline & MM & 435.0 & 144.08 & 33.12 & 175.800 & 1904.50 & 13.22 & 4.38 \\
\hline & MA & 442.5 & 140.00 & 31.64 & 177.300 & 2068.50 & 14.78 & 4.67 \\
\hline & $\mathrm{AB}$ & 435.0 & 153.75 & 35.34 & 178.910 & 2236.38 & 14.55 & 5.14 \\
\hline & $\mathrm{AM}$ & 442.5 & 172.50 & 38.98 & 178.570 & 2232.13 & 12.94 & 5.04 \\
\hline & AA & 450.0 & 168.75 & 37.50 & 178.600 & 2232.50 & 13.23 & 4.96 \\
\hline
\end{tabular}

De las Figura 4 y 5 se demuestra que la potencia eléctrica consumida por el sistema operando con R-134a, es menor que la potencia eléctrica consumida operando con
$\mathrm{R}-12$, para todas las condiciones de carga del evaporador y condensador; ello utilizando válvula termostática o tubo capilar en el proceso de expansión. 


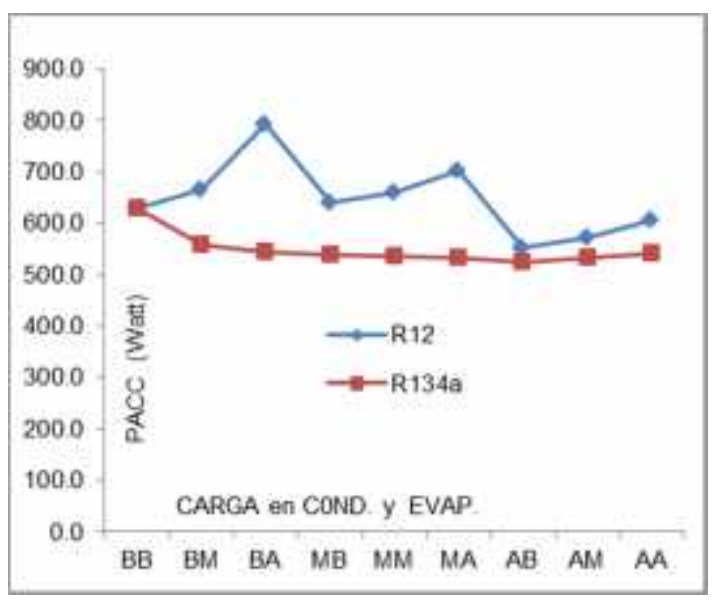

Fig. 4 Potencia consumida vs cargas en condensador y evaporador.

Expansión con válvula termostática.

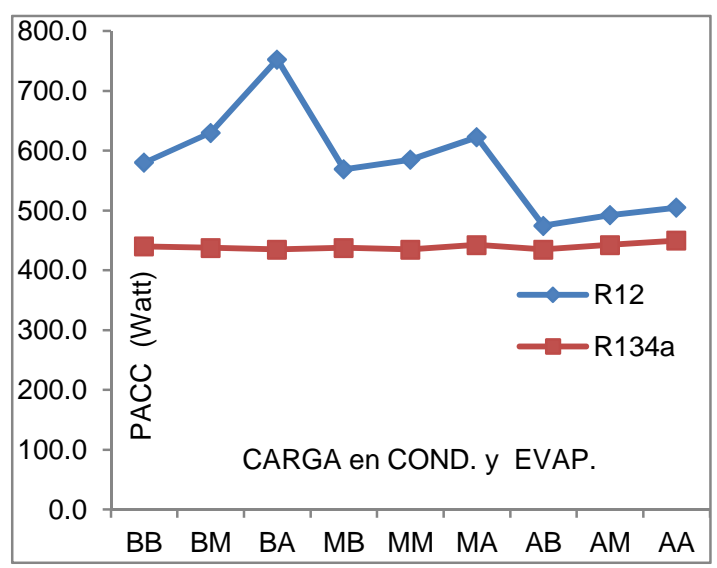

Fig. 5 Potencia consumida vs cargas en condensador y evaporador.

Expansión con tubo capilar.

En las Figuras 6 y 7 se observan los comportamientos de las eficiencias del motor- compresor en función de las cargas en condensador y evaporador, donde se demuestra el mayor rendimiento alcanzado cuando el sistema opera con refrigerante ecológico R-134a, frente a la operación con $\mathrm{R}-12$, bien funcionando con válvula termostática o bien con tubo capilar.

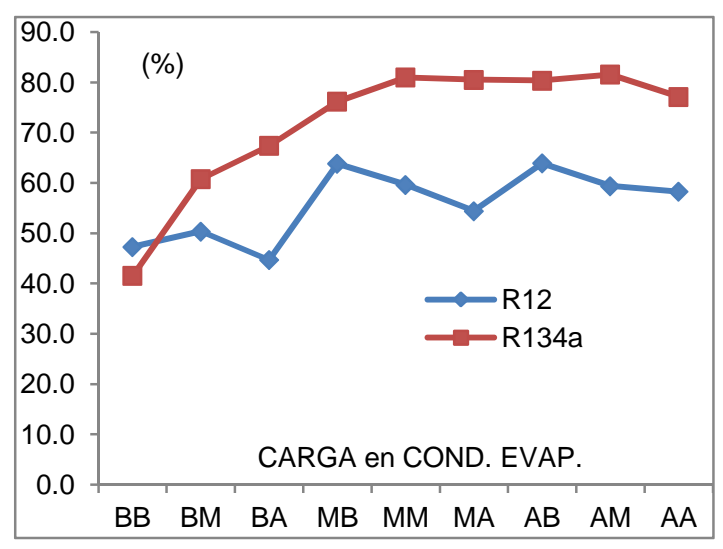

Fig. 6 Eficiencia del motor-compresor, operando con expansor válvula termostática. 


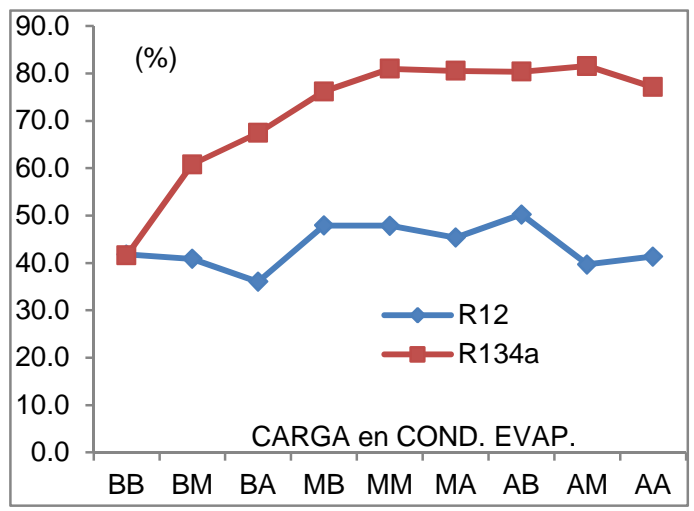

Fig. 7 Eficiencia del motor-compresor, operando con expansor tubo capilar.

Otro de los indicadores de la mayor capacidad de refrigeración que se logra utilizando R-134a, es el Efecto Refrigerante Útil, el mismo que a lo largo de la performance de operación, supera a los valores alcanzados operando con R-12 (ver Figuras 8 y 9. Como elementos de corroboración de resultados se tienen los Coeficientes de Performance tanto del ciclo
(Figuras 10 y 11) como del sistema (Figuras 12 y 13); estos son mayores cuando el sistema trabaja con refrigerante ecológico R-134a, frente a cuando el sistema opera con R-12, a lo largo de todas las condiciones de carga sometidos al condensador y evaporador, así como utilizando válvula termostática o tubo capilar en el proceso de expansión.

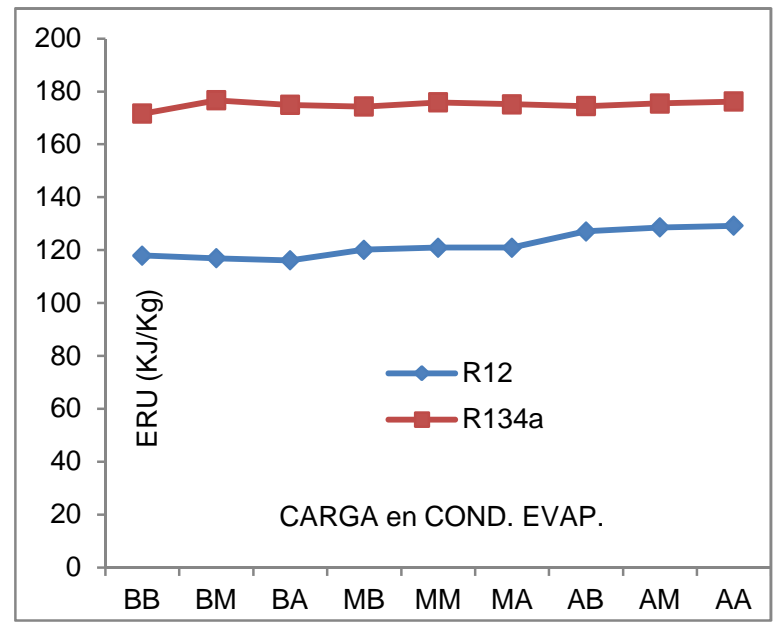

Fig. 8 Efecto refrigerante operando con válvula termostática.

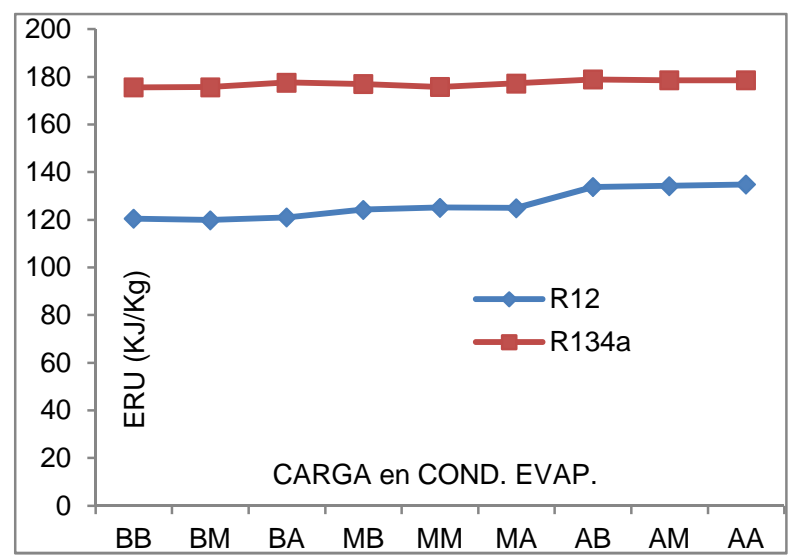

Fig. 9 Efecto Refrigerante operando con tubo capilar. 


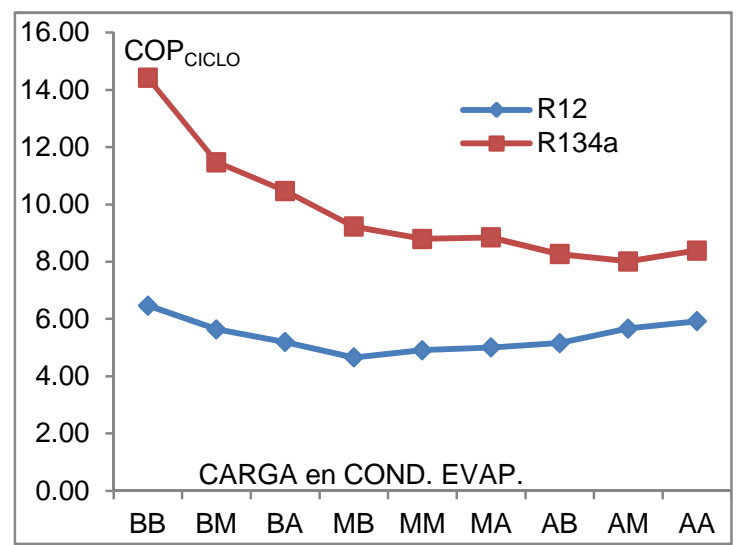

Fig. 10 Coeficiente de performance del ciclo operando con válvula termostática.

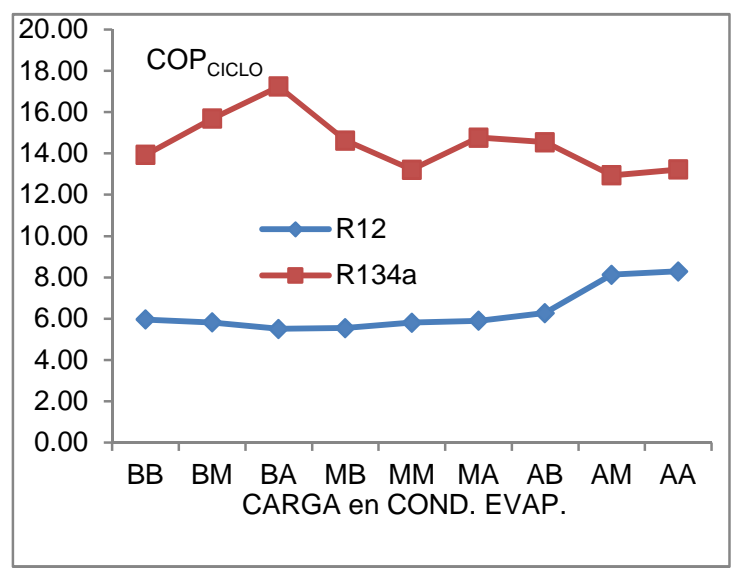

Fig. 11 Coeficiente de performance del ciclo operando con tubo capilar.

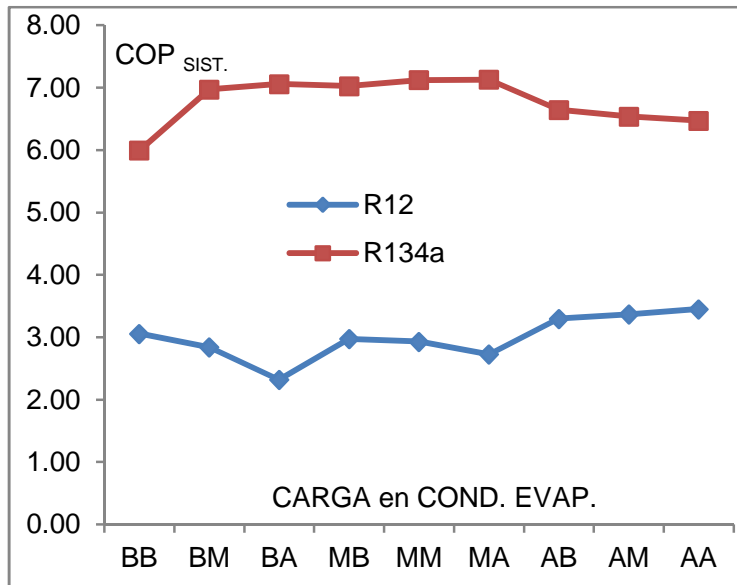

Fig. 12. Coeficiente de performance del sistema operando con válvula termostática. 


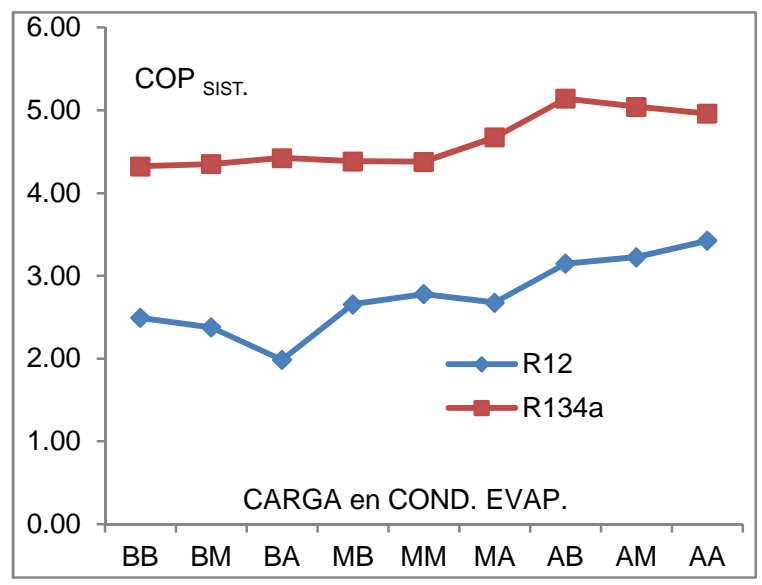

Fig. 13 Coeficiente de performance del sistema operando con tubo capilar.

En la Figura 14 se muestra un diagrama real presión-entalpia representativo de la performance que desarrolla el sistema de refrigeración, operando tanto con R-12 como con R-134a para similares condiciones externas exigidas al evaporador y condensador. De ello se demuestra que la potencia térmica de compresión es similar e incluso menor operando con R-134a frente a la operación con R-12, manteniéndose en los mismos niveles de presión, lo cual es indicativo de confiabilidad de trabajo y vida útil del compresor de origen alimentado con R-12.

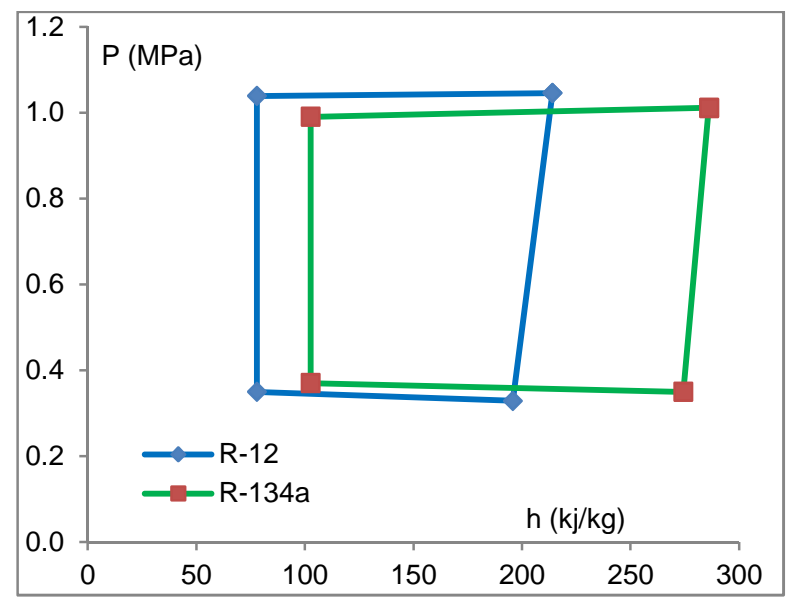

Fig. 14 Diagrama P-h real.

\section{CONCLUSIONES}

Se demuestra que el comportamiento del sistema de refrigeración experimental, posee una mejor performance energética cuando se utiliza el refrigerante ecológico $\mathrm{R}-134 \mathrm{a}$, respecto a los resultados de su operación con el R-12, en el amplio margen de cargas exigidas al evaporador $y$ condensador.

El rendimiento del proceso de compresión y la estabilidad de flujo en el proceso de expansión; alcanzan mayores y mejores condiciones cuando el sistema opera con R134a frente a la operación con R-12, para cualquier nivel de carga del evaporador y condensador

Desde el punto de vista de ahorro energético, se demuestra que la potencia activa de consumo del sistema de refrigeración operando con R-134a, es menor que la potencia activa consumida por el sistema operando con R-12, a lo largo de 
todas las condiciones de carga en el evaporador y condensador.

A las ventajas energéticas, demostradas experimentalmente en el funcionamiento de la refrigeradora operando con R-134a frente al R-12; se suman las ventajas económicas que representa cuando solamente se hace la sustitución del refrigerante. Los costos que representa la conversión propuesta en la investigación; esto es, acondicionamiento del sistema, carga del refrigerante R-134a y dosificación optima del aceite de lubricación, ascienden a 90 USS, lo cual representa menos del $10 \%$ del costo de adquisición de una refrigeradora ecológica nueva, y un $25 \%$ de lo que representa el cambio del compresor diseñado para operación sólo con refrigerante ecológico.

\section{REFERENCIAS}

1. ASHRAE HANDBOOK, "Heating, Ventilating and air Aconditioning Applications. SI Edition, American Society of heating refrigerating and air conditioning engineers", Inc. USA, 1999.
2. GOSWAMI, D. "Selecting a Refrigerant: Comparison Made Easier". USA, 1993.

3. ESCANES, F., PEREZ, C. "Thermal and Fluid -Dynamic Behaviour of Double-Pipe Condensers and Evaporators a Numerical Etudy". Barcelona, 1994.

4. OLIVA, A., PEREZ, C. "Numerical Simulation of a Single Stage Vapor Compression Refrigerating Unit". Universidad Politécnica de Catalunya. 1994.

5. RIGOLA, J., OLIVA, A. "Numerical study of a single stage vapor compression refrigerant unit using noncontaminant refrigerants" - Universidad Politécnica de Catalunya, 1996.

6. KOELET, P. C. "Precisiones sobre la utilización de los fluidos frigorígenos". Belgium, 2000.

7. CENGEL, Y., BOLES, M. "Termodinámica. McGRAW-Hill", 2000.

Correspondencia: salome@uni.edu.pe

Recepción de originales: enero 2014 Aceptación de originales: abril 2014 


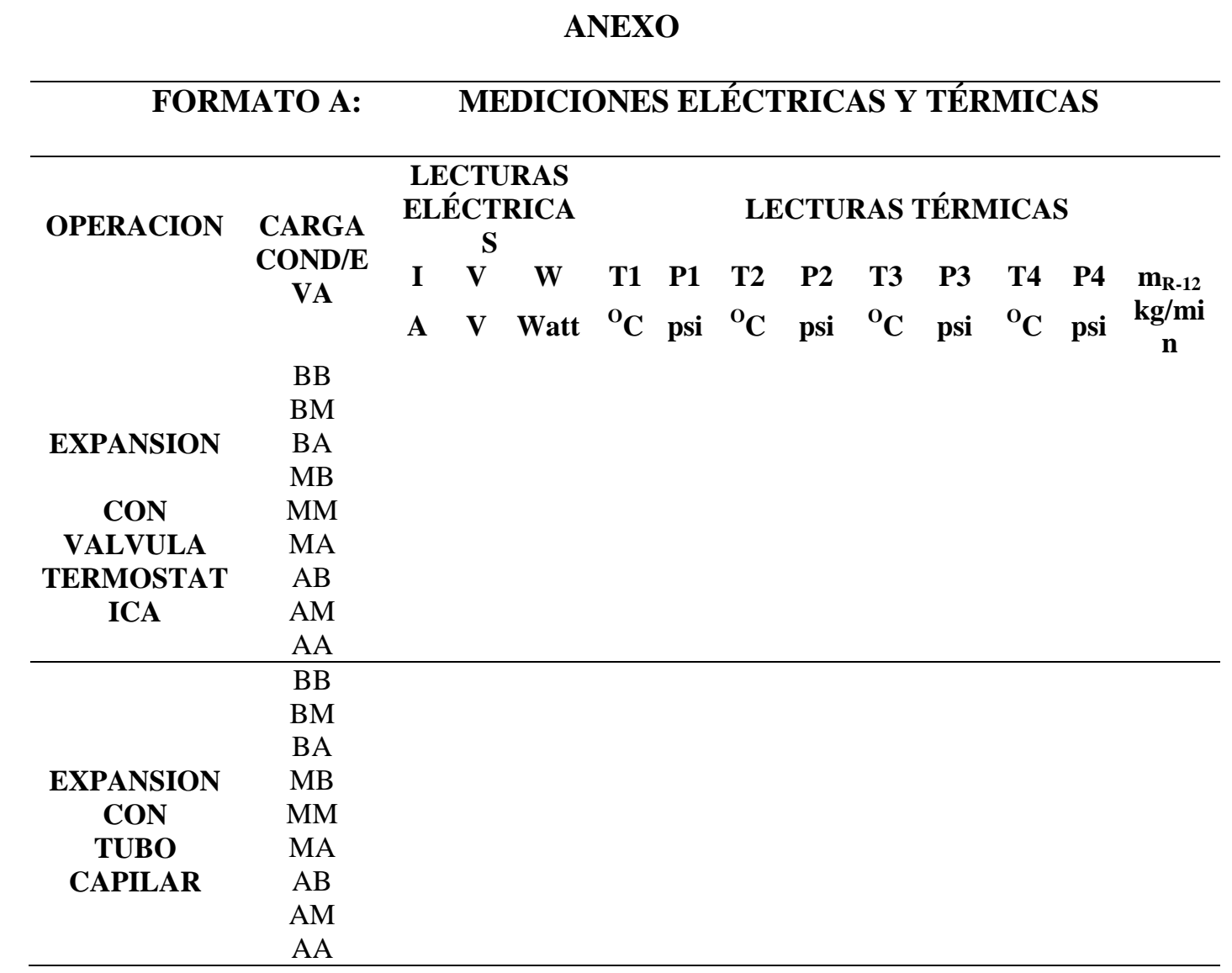

FORMATO B: MEDICIONES EN LAS CARGAS

\begin{tabular}{|c|c|c|c|c|c|c|c|c|}
\hline \multirow[b]{2}{*}{ OPERACIÓN } & & \multicolumn{3}{|c|}{ CARGA CONDENSADOR } & \multicolumn{4}{|c|}{ CARGA EVAPORADOR } \\
\hline & $\begin{array}{c}\text { CARGA } \\
\text { COND/EVA }\end{array}$ & $\begin{array}{cc}\text { Wvi } & \text { TD } \\
\text { Watt } & { }^{\circ} \mathrm{C}\end{array}$ & $\begin{array}{l}\text { TP } \\
{ }^{\circ} \mathrm{C}\end{array}$ & $\begin{array}{c}\mathbf{v} \\
\mathbf{m} / \mathbf{s}\end{array}$ & $\begin{array}{l}\text { Wvd } \\
\text { Watt }\end{array}$ & $\begin{array}{l}\text { TD } \\
{ }^{\circ} \mathrm{C}\end{array}$ & $\begin{array}{l}\mathbf{T P} \\
{ }^{\mathbf{0}} \mathrm{C}\end{array}$ & $\begin{array}{c}\mathbf{v} \\
\mathbf{m} / \mathbf{s}\end{array}$ \\
\hline \multicolumn{9}{|c|}{ BB } \\
\hline \multicolumn{9}{|c|}{ BM } \\
\hline \multicolumn{9}{|l|}{ EXPANSION } \\
\hline \multicolumn{9}{|c|}{ MB } \\
\hline \multicolumn{9}{|l|}{ CON } \\
\hline \multicolumn{9}{|l|}{ VALVULA } \\
\hline \multicolumn{9}{|l|}{ TERMOSTATICA } \\
\hline \multicolumn{9}{|c|}{$\mathrm{AM}$} \\
\hline \multicolumn{9}{|c|}{ AA } \\
\hline \multicolumn{9}{|c|}{ BB } \\
\hline \multicolumn{9}{|c|}{ BM } \\
\hline \multicolumn{9}{|c|}{ BA } \\
\hline \multicolumn{9}{|l|}{ EXPANSION } \\
\hline \multicolumn{9}{|l|}{ CON } \\
\hline \multirow{2}{*}{\multicolumn{9}{|c|}{ TUBO CAPILAR }} \\
\hline & & & & & & & & \\
\hline \multicolumn{9}{|c|}{$\begin{array}{l}\mathrm{AB} \\
\mathrm{AM}\end{array}$} \\
\hline \multicolumn{9}{|c|}{ AA } \\
\hline \multirow{2}{*}{\multicolumn{3}{|c|}{$\begin{array}{l}\text { TEMPERATURA DE BULBO SECO, TBS: } \\
\text { TEMPERATURA DE BULBO HUMEDO,TBH: }\end{array}$}} & ${ }^{\circ} \mathrm{C}$ & & & & & \\
\hline & & & ${ }^{\circ} \mathrm{C}$ & & & & & \\
\hline
\end{tabular}

